\title{
Dynamicity of exosomes as immuno-oncological biomarkers in secondary metastasis and cancer therapy
}

\author{
Sraddhya Roy ${ }^{1}$, Ananya Das ${ }^{1}$, Nushrat Jahan ${ }^{1}$, and Nabanita Chatterjee ${ }^{1}$ \\ ${ }^{1}$ Chittaranjan National Cancer Institute
}

June 15, 2020

\begin{abstract}
Being a tiny component, all the intra-cellular orchestra starting from exosome generation till its escape from the immune cells, is connected with biogenic dynamicity of molecular signaling in the cells. The biogenesis of exosomes follows the endocytic pathway thereby releases the intraluminal vesicles in the extracellular space. Exosomes play critical role in oncogenic signaling in the primary tumor microenvironment as well as distant sites to make a pre-metastatic niche (PMN) for the tumor cells to cause future metastasis. Moreover, tumor derived exosomes (TDEs) play an important role in invasion-metastasis cascade by initiating paracrine and autocrine signals in the tumor milieu thus activating the epithelial-mesenchymal transition (EMT) in the neoplastic epithelial cells. The exosomes taken up by the distally situated organ tissues facilitates the formation of pre metastatic niche allowing the tumor cells to arrive, extravasate and eventually colonize in the new microenvironment and thereby promoting distant metastasis (The Seed and Soil Theory). Importantly, secondary metastasis is also achieved by altering the extracellular matrix by the TDEs as well as by escaping the immune surveillance, which in turn modulate the host immunity and gradually results in the disease progression. Exosomes exhibiting the properties like high target specificity combined with low immunogenicity and biocompatibility aids the exosomes to sow its seed in the new field of therapies and thus all the remarkable properties make it a focus of interest in the research field.
\end{abstract}

\begin{abstract}
Being a tiny component, all the intra-cellular orchestra starting from exosome generation till its escape from the immune cells, is connected with biogenic dynamicity of molecular signaling in the cells. The biogenesis of exosomes follows the endocytic pathway thereby releases the intraluminal vesicles in the extracellular space. Exosomes play critical role in oncogenic signaling in the primary tumor microenvironment as well as distant sites to make a pre-metastatic niche (PMN) for the tumor cells to cause future metastasis. Moreover, tumor derived exosomes (TDEs) play an important role in invasion-metastasis cascade by initiating paracrine and autocrine signals in the tumor milieu thus activating the epithelial-mesenchymal transition (EMT) in the neoplastic epithelial cells. The exosomes taken up by the distally situated organ tissues facilitates the formation of pre metastatic niche allowing the tumor cells to arrive, extravasate and eventually colonize in the new microenvironment and thereby promoting distant metastasis (The Seed and Soil Theory). Importantly, secondary metastasis is also achieved by altering the extracellular matrix by the TDEs as well as by escaping the immune surveillance, which in turn modulate the host immunity and gradually results in the disease progression. Exosomes exhibiting the properties like high target specificity combined with low immunogenicity and biocompatibility aids the exosomes to sow its seed in the new field of therapies and thus all the remarkable properties make it a focus of interest in the research field.
\end{abstract}

Keywords: Exosome, Secondary metastasis, Tumor microenvironment, Immune-modulation, Drug resistant, Anti-cancer therapy

Abbreviations: 
Abs: Apoptotic blebs; ABC:ATP binding cassette; ADCC: Antibody dependent cell cytotoxicity; Ago: Argonaute; AKT: Protein Kinase B; ALG2: Apoptosis linked gene 2; ALIX: ALG-2-interacting protein X; AMSH: Associated molecular with SH3 domain of STAM; Ang- Angiopoeitin; APC: Adenomatosis polyopsis coli; ARG: Arginase; ATF: Activating transcription factor; BMDCs: Bone marrow derived cells; BMP: Bone morphogenic protein; CAFs: Cancer associated fibroblast; CAM: Cell adhesion molecules; CCL: C-C motif chemokine ligand; CD: Cluster of differentiation; CHMP: Charged multivesicular body protein; CHD: Chromodomain-helicase-DNA-binding protein; CME: Clathrin mediated endocytosis; CRP: C- reactive protein; CXCL: C-X-C motif chemokine; DCs: Dendritic cells; DUBs: Deubiquinating enzymes; EIPA: Ethylisopropyl amiloride; EMT: Epithelial- mesenchymal transition; EphB: erythropoietin-producing human hepatocellular B; Ephrins: Eph receptor-interacting proteins; ERK: Extracellular signal regulated kinase; ESCRT: Endosomal sorting complex required for transport; ETS: Avian erythroblastosis E26 homolog 1; EVs: Extracellular vesicles; exo-AnxA2: Exosomal Annexin A2; FasL: Fas ligand; FGF: Fibroblast growth factor; GCSF: Granulocyte colony stimulating factor; HCC: Hepatocarcinoma cells; HCT: Human colon adenocarcinoma; HGF: Hepatocyte growth factor; hnRNP: Heterogenous nuclear ribonucleo protein; HNSCC: Human head and neck squamous cell carcinoma; HPCs: Hematopoetic progenitor cells; HSPGs: Heparan sulfate proteoglycans; HSPs: Heat shock proteins; ICAM: Intercellular adhesion molecule 1; IL:Interleukin; ILK: Integrin linked kinase; ILVs: Intraluminal vesicles; kDa: Kilo Dalton; LAMP: Lysosomal associated membrane protein; LBPA: Lyobisphosphaditic acid; LDL: Low density lipoprotein; LEs: Late endosomes; LFA: Lymphocyte function- associated antigen; Lnc: Long non-coding; LNP: Lipid nanoparticle; M1/2: Macrophage 1/2; MAC: Membrane attack complex; MCs: Myeloid cells; MDR: Multiple drug resistant; MEK: Mitogen Activated Protein Kinases; MET: Mesenchymal- epithelial transition; MHC: Major histocompatibilty complex; miRNA: Micro Ribonucleic Acid; Mo-MDSCs: Monocyte myeloid derived suppressor cells; mRNA: Messenger Ribonucleic Acid; MSC: Mesenchymal stem cell; MTA: Metastasis-associated protein; mTOR: Mammalian target of rapamycin; MVBs: Multi vesicular bodies; NF: Nuclear factor; NK cells: Natural killer cells; NKG2D: Natural killer group 2D; NO: Nitric oxide; NSF: N-ethylmaleimide sensitive factor; nSMase: Neutral Sphingomyelinase; PAR1: Protease activated receptor; PCNA: Proliferating cell nuclear factor; PDGF: Platelet derived growth factor; PDZ: post synaptic density protein (PSD95), Drosophila disc large tumor suppressor (Dlg1), and zonula occludens-1 protein (zo-1); P-gp: P-glycoprotein; PH: Pleckstrin homology; PI3K: Phosphatidyl inositol 3 kinase; PLP: Proteolipid protein; PMN: Premetastatic niche; RAC: Ras-related C3 botulinum toxin substrate; RANTES: Regulated on Activation, Normal T cell Expressed and Secreted; RISC: RNA induced silencing complex; ROCK: Rho associated coiled coil containing protein kinase; ROS: Reactive oxygen species; S1P: Sphingosine 1 phosphate; SARS: Seryl- tRNA synthetase; SCF: Stem cell factor; SDC: Syndecan; SMVs: Shedding microvesicles; SNAP: Soluble NSF-attachment protein; SNARE: N-ethylmaleimide sensitive factor attachment protein receptor; Sphk: Sphingosine phosphate kinase; STAM: Signal transducing adaptor molecule; STAT: Signal transducer and activation of transcription; SUMO: Small ubiquitin like modifier protein; TAMs: Tumor associated macrophages; TANs: Tumor associated neutrophils; Tc cells: T cytotoxic cells; TCR: T cell receptor; TDEs: Tumor derived exosomes; TDEs: Tumor derived exosomes; TDSFs: Tumor derived secreted factors; TERF: Telomeric repeat binding factor; TFs: Transcription factors; TGF: Transforming growth factor; Th cells: T helper cells; THBS: Thrombospondin; TLR: Toll like receptor; TNF (R): Tumor necrosis factor (receptor); tPA: Tissue plasminogen activator; TRAIL: TNF related apoptosis inducing ligand; Tregs: T regulatory cells; TSG: Tumor susceptibility gene; UBPY: Ubiquitin isopeptidase Y; uPA: Urokinase-type plasminogen activator; UTR: Untranslated region; VAMP: Vesicle associated membrane protein; VEGF: Vascular epithelial growth factors; Vps: Vacuolar protein sorting; VTA: Ventral tegmental area

\section{Introduction}

The intercellular communications were previously thought to be mediated by ligand receptor interactions and direct cell to cell contacts. With gradual increase in the interest of research, it is discovered that there exist another mode of interaction between cells conducted by membranous extracellular vesicles (EVs) (Théry et al., 2002; Mathivanan et al., 2010). EVs are categorized into three main categories namely exosomes, shedding micro vesicles (SMVs) or ectosomes and apoptotic blebs (Abs) (Gangoda et al., 2015). Despite of having 
similarities, difference lies in mode of formation, variation in sizes and buoyant density (Mathivanan et al., 2010). Ectosomes $>100 \mathrm{~nm}$ and are released from variety of cells when outward protrusion occurs followed by pinching of the cytoplasmic content contained vesicles from the plasma membrane (Cocucci et al., 2009) and $\mathrm{Abs}(>0.2 \mu \mathrm{m})$ are condensed remains of the apoptotic cells (Hristov et al., 2004) being released by the dying cells (Théry et al., 2009). The exosomes first noted as microvesicles released by neoplastic cells with 5 nucleotidase activity(Trams et al., 1981) but the microvesicles are of endocytic origin were proved when their involvement in combing out of transferrin receptors in the later stages of the process of maturation of reticulocytes was observed (Rajagopal and Harikumar, 2018). Exosomes, biocompatible nanoparticles (30-150nm) secreted by almost all cells, and ubiquitously present in all bodily fluids (blood, urine, saliva, semen, cerebrospinal fluid, plasma, amniotic fluid etc) (McKelvey et al., 2015), carrying original specific cargoes which makes them perform various vital functions in the body (Rajagopal and Harikumar, 2018), locally or distantly, secreting soluble factors(McKelvey et al., 2015). Delicate researches have focused on the functions of exosomes likely by affecting the body by manipulating the immune system and marking their presence in several therapies. Exosomes participate in the process of EMT and causing secondary metastasis. The different molecules or cargoes that are carried by the exosomes both on their surface as well as inside the vesicles are responsible for functionality of exosomes. Furthermore, exosomes are also used as drug delivery vehicles. Epidemiological study supports that EV implied pivotal role for identification of diseases occurrences by patients' biofluids (Verma et al., 2015).

\section{Biogenesis of exosomes}

\section{Origin}

The internalization of various cellular components by invagination of plasma membrane in clathrin or caveolin dependent manner (Xu et al., 2018), and the process of forming vesicles, is known as endocytosis (Huotari and Helenius, 2011). Firstly, the vesicles formed as early endosomes followed by the biochemical modulations to form the late endosomes (LEs) or considered as multivesicular bodies (MVBs), and intraluminal vesicles (ILVs). These MVBs could be the precursors of exosomes (Kim et al., 2018) as they secrete the ILEs outside the cells, as the exosomes. The early endosomes are involved in sorting the cellular components whereas the late endosomes exhibit the properties of MVBs and can either be degraded or gets assembles in Golgisome (Wan et al., 2018). The signal for undergoing the path of degradation is the ubiquitylation of the cargoes being loaded in the ILVs (Williams and Urbé, 2007). Cytosolic proteins and nucleic acids are uptaken in the ILVs, in a selective and non-selective manner and one of such process is microautophagy (Huotari and Helenius, 2011). The MVBs have a two destined fate, either they undergo degradation or secretion (Xu et al., 2018) . Generally MVBs that are rich in cholesterol are destined to fuse with the membrane of the cell to be released out and the cholesterol deficient ones undergoes the lysosomal degradation process (McGough and Vincent, 2016). As they incorporate the cellular components during the maturation of LEs, the exosomes reflect the nature of the cell from which it has originated.

\section{Generation}

The exosome generation mainly occurs through two distinct pathways: (i) Endosomal sorting complex required for transport dependent pathway (ESCRT dependent) and (ii) Endosomal sorting complex independent pathway (ESCRT independent) (Figure 1).

\section{ESCRT dependent pathway}

The ESCRT apparatus comprises of four subunits - ESCRT 0, -I, -II and -III which are loaded on the endosomal membrane. The protein sorting in the ubiquitin dependent pathway involves all the subunits of the complex ESCRT 0 is loaded first as it recognizes the mono-ubiquitinated cargoes via its subunits. It then recruits the ESCRT I subunit, which in turn recruits the ESCRT II subunit to the endosomal membrane and both help the ESCRT 0 subunit to bind to the ubiquitinated cargoes with high specificity and impels the endosomal membrane to deform, constrict and invaginate, resulting in release of vesicles in to the endosome. The ILVs with bound ubiquitinated cargoes, that are released into the endosomes undergo the lysosomal degradation unless they are deubiquinylated by the DUBs (Mashouri et al., 2019). In mammals, a ubiquitin 
isopeptidase named AMSH performs deubiquitination and another enzyme named UBPY maintain ubiquitin levels in the cell (Henne et al., 2011). In ubiquitin free mechanism, ALIX protein binds directly with the CHMP4 subunit of ESCRT III complex and incorporates deubiquitinated cargoes to the ILVs. ALIX binds to GPCR- PAR1 and syntenin which in turn binds to syndecans and tetraspanin CD63, thereby sorting all these components as cargoes to the ILVs without any ubiquitylation (McGough and Vincent, 2016). Disassembly of ESCRT complex mediated by ATPase Vps4 and VTA1 can terminate the pathway and recycles the ESCRT subunits for next cycles (McGough and Vincent, 2016).

\section{ESCRT independent pathway}

Depletion of the ESCRT complexes but presence of MVBs throws a light on the fact the biogenesis of MVBs can also take place in the absence of the ESCRT complexes (Stuffers et al., 2009) which is conducted by lipids and tetraspanins (Kim et al., 2018). The trafficking of PLP aids in elucidating the pathway. The endosomal membrane possess certain microdomains rich in sphingolipids that forms ceramide by hydrolytically removing the phosphocholine moiety of sphingomyelin aided by sphingomyelinases and inhibition of sphingomyelinases secreted less exosomes (Trajkovic et al., 2008). The cone shaped structure of the ceramide deforms the endosomal membrane which sorts the PLPs leading to abscission of vesicles to the endosomal compartment (Trajkovic et al., 2008).

\section{Exosomal cargo sorting}

Exosomes being bioactive molecules harbors a variety of molecules on its membrane like lipids, proteins and nucleic acids (DNA, RNA, lncRNAs, mRNA, miRNA). Table 1 (Whiteside, 2015; Li et al., 2017; Reclusa et al., 2017; Sumrin et al., 2018; Wang et al., 2018a; Yousafzai et al., 2018; Huang and Deng, 2019; Jalalian et al., 2019; Meng et al., 2019) provides an account on cancer specific proteins and nucleic acids, respectively, carried as cargoes by the exosomes. The cytosolic proteins being uploaded are tubulin, actin, cofilin; the actin binding proteins as well as the proteins which are engaged in membrane fusion and transport - Annexins and Rab proteins (Théry et al., 2002). Enzymes like peroxidases, enolase-I and molecules like 14-3-3, heterotrimeric G protein, Gi2 $\alpha$ and HSPs are loaded into the interior of the exosome (Théry et al., 2002). Exosomes possess MHC class proteins (Rajagopal and Harikumar, 2018) and tetraspanin proteins (CD9, CD63, CD81, CD82) are on the exosomal surface. Both mRNAs and miRNAs are loaded as exosomal cargo where the mRNA content differs from the parent cell while the miRNA content remains the same (Rajagopal and Harikumar, 2018). The uploading of miRs depends on a specific shuttle sequence GGAG. A SUMOylated hnRNPA2B1 specifically binds to the miRs with GGAG sequence, controlling their upload into the exosome (VillarroyaBeltri et al., 2013). Exosomal mRNAs, are rich in 3 UTR region depicting that it may sort mRNA into the exosomes (Hessvik and Llorente, 2018). The fact that the exosomal membrane is rich in cholesterol, sphingolipids and glycosphingolipids comparison to parent cells, depicts that lipids components may help in cargo sorting and signaling too (Lingwood and Simons, 2010; Record et al., 2014). CD63, CD81, floitilin are sorted by S1P which is formed by the phosphorylation of sphingosine by Sphk1 and Sphk2 and inhibition of this signaling reduces CD63 signals on exosomes as it limits the size of exosomes suggesting involvement of S1P in cargo sorting (Kajimoto et al., 2013).

\section{Exocytosis of exosomes}

As described earlier DUBs in ESCRT dependent pathway enables exosomes to get released but a post transcriptional modification, ISGylation of the TSG101 limits the secretion by directing the MVBs towards lysosomal degradation (Villarroya-Beltri et al., 2016). RabGTPases, the largest family of small GTPases, regulates their secretion, although their mechanism is still uncovered. Investigation shows that the expression of cortactin accompanied by Rab27 and coronin1B can regulate the release of the exosome (Sinha et al., 2016). Silencing of five RabGTPases namely Rab2b, Rab5a, Rab9a, Rab27a and Rab27b by shRNA minimized exosome secretion whereas silencing of Rab6 does not affect exosome secretion level (Ostrowski et al., 2010). Rab27a and Rab27b is keenly involved in exosome and their decrease also accounts for lesser exosome release but with the unchanged protein content of the cell (Blanc and Vidal, 2018). Both Rab27a/b are also involved in determining the size of the MVBs (Blanc and Vidal, 2018). The docking of the MVBs to 
the plasma membrane is also regulated by several Rab effector molecules namely synaptotagmin- $4 / 7\left(\mathrm{Ca}^{2+}\right.$ dependent phospholipid binding protein), exophilin-5(Slac2B), granuphilin-a/b (Slp4) whereas the fusion event is regulated by Rab11, Rab31, R-SNARE protein YKT6, three members of t-SNARE protein family (SNAP23, syntaxin3, syntaxin4), v-SNARE protein family (VAMP 7/ VAMP 8/ TI-VAMP) (Théry et al., 2002; McKelvey et al., 2015; Yousafzai et al., 2018). Some soluble factors like NSF and SNAP are important for the fusion of intracellular fusion events (Théry et al., 2002). Rab11 and Rab35 participate in both exosome release and recycling the membrane components (Blanc and Vidal, 2018).

Internalization of exosomes

The internalization of exosomes begins with adhesion of primary cells where the protein - protein interactions play a vital role through endocytosis (Villarroya-Beltri et al., 2013). CAMs are grouped under five categories namely cadherins, immunoglobulins, a lectin family subclass called selectin, mucin and integrins, differences among them lies in their mode of interaction (Gonda et al., 2019). The presence of tetraspanins, integrins, proteoglycans and lectins on the exosomal membrane facilitates their interactions with the recipient cells (Mulcahy et al., 2014). Exosomal integrins and immunoglobulins mediate the interaction between cells and participate in exosomal uptake by dendritic cells, macrophages, T cell (Villarroya-Beltri et al., 2013; Hessvik and Llorente, 2018). In CD4 ${ }^{+}$cells, exosomes are internalized via interaction between TCR- MHC and LFA1 -ICAM1. Heparan sulfate proteoglycans (HSPGs) with attached sulfated glycoaminogly can polysaccharides facilitates exosomes adherence. Heparinase treatment on EVs did not affect their uptake suggesting that HSGPs on the cell surface contribute to internalization (Christianson et al., 2013). Syndecan and glycipan are the two main types of HSPGs that function in (i) cell adherence, motility exosomal binding, content delivery and (ii) role in endocytosis for delivery of the ligands (Villarroya-Beltri et al., 2013). HSPGs act as fibronectin receptor when it binding to fibronectin either present on exosome or on the target cells(Purushothaman et al., 2016). Lectins function in recognizing and binding to glycan moieties. Lectins are categorized under (i) the transmembrane C-type lectins and selectins, (ii) sialic acid binder named Siglecs and (iii) a galactoside binder named galectins (Villarroya-Beltri et al., 2013). C-type lectins (DC-SIGN, DEC-205, MUC16 ) and galectin (Galectin-5) are related with EVs adhesion (Hessvik and Llorente, 2018).

The cellular responses induced by membrane bound or soluble FasL, TRAIL or TNF do not require the internalization of exosomes rather they are cleaved by MMPs to form soluble cytokines so that they can adhere to the receptors of the target cells for juxtacrine or soluble signaling (McKelvey et al., 2015). Soluble signaling involves proteolytic cleavage of the ligands where as juxtacrine signaling requires juxtaposition of the exosomal ligands and receptors on the recipient cells (Su et al., 2018). Fusion between the exosomes and the recipient cell occurs when the vesicles fuses with the plasma membrane of the recipient cell forming hemi-fusion stalk that leads to a fusion pore formation, through which its content is released (McKelvey et al., 2015)(Hessvik and Llorente, 2018). Fusogenic lipids like phosphatidic acid, BMP on the exosomal membrane causes "lipid interdigitation", facilitating fusion(Villarroya-Beltri et al., 2013).

Endocytosis mechanisms responsible for internalization are (i) macropinocytosis, (ii) phagocytosis, (iii) clathrin mediated endocytosis, (iv) caveolin mediated exocytosis and (v) lipid raft mediated endocytosis. Micropinocytosis gives rise to micropinosomes by uptaking extracellular fluids (Villarroya-Beltri et al., 2013). Attenuation of the $\mathrm{Na}^{+}-\mathrm{H}^{+}$ion exchange activity and PI3K pathway by inhibitors like EIPA results in the decreased uptake of exosomes suggesting that macropinocytosis relies on $\mathrm{Na}^{+}$and PI3K, with involvement of Rac1, Ras and Src (McKelvey et al., 2015; Hessvik and Llorente, 2018). TDEs are internalized by phagocytic cells by phagocytosis whereas the TDEs remain on the bound to the surfaces of the non-phagocytic cells, thus they are internalized more effectively by phagocytic cells than by non-phagocytic cells (Feng et al., 2010). CME involves a protein clathrin that form a cage like structure around the exosome which invaginates the plasma membrane of the recipient cells, matures and pinches off to form a vesicle bud followed by shedding off the clathrin protein and fusing with the endosome where the vesicles (120nm) empties all its content. Caveolin mediated endocytosis is similar to CME but the protein caveolin coats vesicle of smaller size, even less than $60 \mathrm{~nm}$, , aided by insulin and albumin, which are present on the exosomal surface (Villarroya-Beltri et al., 2013, 2016). Lipid rafts are composed of protein receptors, cholesterol and glycosphingolipids that float 
in aggregated form and is assisted by flotillins aiding in exosomes uptake in clathrin/caveolin independent mode (Hessvik and Llorente, 2018). Histones proteins on the exosomal surface interact with cell surface protein syndecan-4 (SDC4) facilitating exosome internalization (Ochieng et al., 2018).

\section{Exosomes mediated secondary metastasis}

The entire mechanisms that promote tumor cells growth, proliferation and spread can be summed up in a word "metastasis" and is a potent cause for mortality from cancer. Metastasis begins when the primary tumor cells starts to disseminate from the local site and migrate to a distantly located site and since the exosomes play a crucial role in the intercellular crosstalk, they mark themselves in promoting metastasis. TDEs come into play their role at every stage of the process of metastasis. The stages that are involved in the process of metastasis have been schematically represented in Figure 2.

Exosomes in initiation of Epithelial-Mesenchymal transition

EMT can be broadly defined as a process that comprises of molecular changes, minimized cell recognition and adhesion, thereby promoting cell motility (Heerboth et al., 2015). During EMT the cancer cells lose their epithelial attributes and gain mesenchymal characters, which are required for dissemination, invasion and metastasis (Conigliaro and Cicchini, 2018). The gain of mesenchymal attributes is due to crosstalk between cells, where exosomes comes into action (Vella, 2014a). Exosomes concentration released from transformed cells is greater compared to untransformed cells, with increased mesenchymal markers like vimentin, $\mathrm{N}$-cadherin, fibronectin, decreased epithelial markers like E-cadherin, EpCam, occludins, etc. and increased EMT inducing TFs like ZEB1, SIP1/ZEB2, Twist1/2, Snai1/2/3 (Snail/Slug/Smuc), which mainly suppresses of epithelial genes and reprogram gene expression during EMT/MET plasticity (Vella, 2014b; Conigliaro and Cicchini, 2018). EMT inducers like TGF $\beta$, TNF $\alpha$, IL-6, TSG101, Annexin2, caveolin1, integrin3, ILK1, MMPs, PDGFs are associated with exosomes. TDEs released under hypoxic condition have increased EMT transducers than those released under normoxic state (Vella, 2014b; Syn et al., 2016). Novel EMT markers like FOX, GATA family, SRY (SOX) family transcription markers, are also reported that work in association with Snai1/Snai2 (Yuan et al., 2014). TDEs induce EMT by activating signaling pathways e.g. HCC cells derived exosomes confer properties of invasion and migration to low metastatic cells by activating MAPK/ERK signaling pathway or by carrying unique protein molecules on their surface which can remodel the recipient cells (Vella, 2014b; Conigliaro and Cicchini, 2018). Moreover the lncRNAs activated by TGF $\beta$ can upregulate ZEB1/ZEB2 expression in HCC cells, thereby inducing EMT (Yuan et al., 2014). OSCC derived TDEs express EGFR on their surface and these TDEs when gets internalized by the epithelial cells induces them to acquire mesenchymal like characteristics (Fujiwara et al., 2018). The exosomal MMPs (mainly MMP-13) activate the Wnt signaling pathway which transcriptionally activate upregulation of the factors responsible for EMT (Blavier et al., 2010). MMP-3 aids in generation of ROS in the cytoplasm that upregulates expression of Snai1 and along with MMP-7 and MMP-14, can cleave the ectodomain of E-cadherin that facilitates cell invasion, whereas MMP-9 participates in increasing the level of mesenchymal markers (Gonzalez-Avila et al., 2019). IGFR, EGFR etc. expressed by exosomes induces EMT in which IGFR upregulates the expression of ZEB, which activates TGF- $\beta$ whereas EGFR participates in phosphorylation of $\beta$-catenin so that it destabilize the tight junction (Heerboth et al., 2015). FAK, a tyrosine kinase molecule can also phosphorylate $\beta$-catenin followed by its translocation to the nucleus to transcribe the target genes responsible for EMT (Heerboth et al., 2015). Exosomal TGF $\beta$ also participates in inducing EMT by activating the Smad dependent pathway resulting in overexpression of Snail inside the nucleus, thereby downregulating E-cadherin(Heerboth et al., 2015). The alterations occurring to a cell during EMT are not genetic mutations rather they are epigenetic changes like histone methylation, histone deacetylation, O-glycosylation because they are reversible and all these changes are induced by the transcription factors generated by the EMT inducers like Snai1, ZEB, Twist which can silence E-cadherin and integrina4 by hypermethylation (Kevin Range, 2012; Heerboth et al., 2015)..

Exosomes in modulation of tumor microenvironment and establishment of pre-metastatic niche

The cellular environment accompanied by tumor cells with other cells like fibroblasts, macrophages, dendritic, 
immune/inflammatory cells is regarded as TME, with other factors like proteins, soluble cytokines and EVs (Rajagopal and Harikumar, 2018). The metabolic crosstalk between the cancer cells and other cells in the TME initiates tumor metastasis. In gastric cancer, CD97 present on the TDEs induce cell proliferation through MAPK signaling (Maia et al., 2018). Among all the cells present in the TME CAFs, stromal fibroblasts and macrophages are important for their function. TDEs can also manipulate the stromal cells in the TME that gastric cancer cell derived exosomes can translocate EGFR in liver stromal cells and can suppress mir-26a/b, thereby activates HGF (Zhang et al., 2017). The macrophages in the TME show plasticity in their phenotype in response to their environmental conditions and TDEs are found to be one of the factors responsible for their polarization in the TME, which are recognized by their Fc receptors (Maia et al., 2018; Rajagopal and Harikumar, 2018). TDEs also elevate the migratory property of the host cells (Blanc and Vidal, 2018). Under hypoxia, miR-21 from OSCC derived exosome induced pre-metastatic senses in normal cells and miR-200c-3p, of OSCC derived exosomes can silence CHD9 and WRN and increase the invasive properties in squamous tongue carcinoma (Zorrilla et al., 2019). TDEs make the TME an absolute habitat for the cancer cells for the interaction with the other cells to perform the normal functions to maintain the pre-metastatic niche and TDSFs, EVs, BMDCs, suppressive immune cells and host stromal cells also enrich the environment (Guo et al., 2019).

The primary tumor cells induces the secretion of soluble cytokines and growth factors (TDSFs like VEGF, $\mathrm{TNF} \alpha$, IL etc.) which influences the process of metastatic by stimulating and recruiting the myeloid cells in a paracrine fashion, thus the migration rate increases towards the target site (Greening et al., 2015a). TDEs also modulate the BMDCs already recruited to the target site to gain a pro-vasculogenic phenotype to influence the metastasis. Extracellular matrix also get demodulated in presence of oncogenic exosomes as they harbor several proteins and MMPs on their surface that facilitates the migrating tumor cells to evade the host stroma of the pre-metastatic niche (Tickner et al., 2014).

Dual role of exosomes in immune modulation

The ways of immune surveillance escape of cancer cells could be altered by exosomal involvements like hypoxic tumor-derived microvesicles facilitate immune escape by blocking the receptors of NK cells and $\mathrm{CD}^{+8}$ (Ludwig and Giebel, 2012). Exosomes shows heterogeneity i.e. metastatic or non-metastatic (Figure 3). Non-metastatic exosomes recruit immune cells to patrol the pre-metastatic niche whereas the metastatic ones mediates immune regulation and recruits immune suppressive cells aided by ROS, NO, ARG1, TNFR-2 in reduced immune surveillance(Guo et al., 2019). TDEs possess tumor specific antigens presentation to DCs (Webber et al., 2015) and direct and cross presentation pattern for $\mathrm{T}$ cell activation. Direct presentation occurs when the exosomes are directly present antigens to T cells via MHC I/II - peptide complexes whereas indirect presentation followed by APCs internalization or fusion of TDEs with APCs. The further processing and expression of antigens in activating $\mathrm{CD} 4^{+}$and $\mathrm{CD} 8^{+} \mathrm{T}$ cells are carried by exosome(Greening et al., 2015b).

The exosome on the mast cell surface derived exosomes present processed antigen peptides to activate DCs and NK cells derived exosomes secretes granzyme B and perforins which mediate cytotoxic effect on the various tumor cells (Othman et al., 2019). TDEs when interact with the monocytes, induces higher secretion of various cytokines like IL-6, IL-8, IL-1 $\beta$, TNF $\alpha$ in activating TFs like NF- $\varkappa$ B and STAT3 to promote immune surveillance escape(Bretz et al., 2013). PD-L1 expression is also directed by TDEs with induction of monocytes to secrete pro-tumorigenic cytokine-chemokine like CCL2, CCL3, CCL4, IL-6, CXCL10 (Haderk et al., 2017). Furthermore a novel paracrine loop has been established in contact with OSCC and associate TAM derived exosome that can induce M1 by transferring THBS1 to the TAMs and activating p38, AKT, SAPK/JNK signaling (Chen et al., 2018). In breast cancer, NF- $x$ B is activated when palmitoylated ligands on TDEs interacts with TLR2 on macrophages and stimulates the secretion of pro-inflammatory cytokines like GCSF, IL-6, IL-8, IL-1b, CCL2, TNF $\alpha$ (Chow et al., 2014). Under hypoxic condition of pancreas cancer, miR-301a-3p of TDEs induces M2 phenotype polarization by activating PTEN/PI3K $\gamma$ signaling(Wang et al., 2018b). In pre-metastatic niche, the normal fibroblast possess reprogramming under the effect of TDEs, and the acidic environment proves fatal for the immune cells to survive in TME. A monocyte myeloid 
derived-suppressor cells phenotype (Mo-MDSCs) is generated when TDEs suppress monocyte maturation and reduces immune surveillance (Domenis et al., 2017). TDEs induce accumulation MDSCs in the TME when they are internalized by the bone marrow precursor cells and switches their differentiation pathway to MDSCs pathway and because TDEs carry PGE2 and TGF $\beta$, they induce the secretion of Cox-2, promoting tumor growth and exosomal Hsp-70 and Hsp-72 also promotes the gathering and stimulation of MDSCs in the TME, which significantly increase the level of pro-inflammatory cytokines like IL-6, TNF $\alpha$, CCL2, VEGF etc. (Othman et al., 2019). MDSCs derived exosomes can induce polarization of M1 to M2 macrophages via IL-2 production (Burke et al., 2014). In case of impairment of innate immunity, TDEs decrease immune surveillance by diminishing ADCC by inhibiting the NK cells cytotoxicity by reducing perforins and granzymes and decreasing MAC formation and cell lysis. Whereas, exosome impairs adaptive immune response by inhibiting $\mathrm{T}$ cell and $\mathrm{B}$ cell function and their differentiation from progenitor cells by inducing apoptosis of the Tc cells due to overexpression of FasL, TNF related apoptosis inducing ligand, CD95L, galectin 9 on exosomal surface and promoting Tregs differentiated from Th cells, induced by TGF $\beta-1$ and IL-10 (Tickner et al., 2014; Steinbichler et al., 2019).

Exosomes in promoting angiogenesis

Angiogenesis can be defined as formation of new blood vessels in an already existing vascular network and activation of the endothelial cells by various angiogenic molecules (Aslan et al., 2019). All the effectors molecules including EphB, VEGF are responsible for angiogenesis, either carrying by the TDEs or the tumor cells themselves. The mechanisms involved in stimulation of angiogenesis by EVs is transfer of miRNA (e.g. miRNA 126/ 214/296/150), proteins like VEGF, FGF-2, PDGF, SCF, RANTES, CRP, MMPs, uPA lipids (S1p) and transcription factors like STAT3, STAT5 and also the activating signaling pathways like PI3K, ERK1/2, Wnt4/ $\beta$-catenin. Whereas the inhibition of angiogenesis is mainly modulated by CD36-dependent uptake of EVs and induction of oxidative stress plus LDL receptor-mediated endocytosis (Todorova et al., 2017). Ang1 contributes to invasion plus survival of endothelial cells whereas Ang2 accompanied by VEGF participates in proliferation and migration of the endothelial cells (Aslan et al., 2019). MMP like MMP-1, -2, $-7,-8,-9,-14$ are actively involved in the process of angiogenesis(Gonzalez-Avila et al., 2019). In addition, Hsp- $90 \alpha$ carried by TDEs also induces the overexpression of MMPs in the pre-metastatic niche (Maxson \& Mitchell, 2016).

TDEs derived from different cells induce angiogenesis in different ways. In endothelial cells, the exosomal integrin $\beta 1$ interacts with the target endothelial cells, conferring proangiogenic effect by activating RAC1ERK1/2-ETS and CCL-2 production (Arderiu et al., 2015). Exosomal $\delta$ like4/Notch signaling also induces blood vessel formation, without having direct cell-cell interaction (Sheldon et al., 2010). Exosomes produces CRP, plasmin, stimulated by VEGF, FGF-2 increases the amount of exosomal MMP-2/9 and transfer of different miRNAs to contribute in the process (Todorova et al., 2017). The transcription of several proangiogenic factors occurs due to the transfer of growth factors like SCF, c-kit, PDGF etc. to the recipient cells. Moreover the miRNAs like mi-R31, mi-R125 elevates the HIF- $\alpha$ secretion and inhibits $\delta$ like4, thereby promoting the tip cell specification, respectively (Todorova et al., 2017).

\section{Role of exosomes in drug resistance}

TDEs mediate drug resistance by: (i) exclusion of the drugs through exosome pathway, (ii) by neutralizing antibody based drugs and (iii) by transfer of miRNAs (Giallombardo et al., 2016). Exosomes secreted from cisplatin resistant ovarian cancer cell contain 2.6 fold more cisplatin than the exosomes released by cisplatin sensitive cancer cells thereby indicating that endocytic compartments involved in exosome pathway act as a vector for the exclusion of the drugs (Safaei et al., 2005). Drugs can also be excluded out via exosomal pathway mediated by ABC transporters (Giallombardo et al., 2016). In neutralizing antibody based drugs, the proteins on TDEs bind to the drugs thereby making them unavailable to the tumor cells to be treated e.g. breast cancer derived exosomes carry HER2, which adheres to trastuzumab thereby nullifying its effect (Ciravolo et al., 2012). Exosomes can reduce ADCC by binding to cancer reactive antibodies (Yousafzai et al., 2018). TDEs transfer of MDR-1/P-gp from resistant to sensitive cells was observed in breast cancer and prostate cancer cells, gaining resistance to docetaxel (Giallombardo et al., 2016). TDEs transferred miRNAs 
render multiple drug resistance in cancer e.g. in cervical cancer resistance to cisplatin is provided by exomiR25. In neuroblastoma, resistivity occurs when the cancer cells release oncomiR-21 which gets internalized by monocytes and these monocytes in turn transcribe oncomiR-155, releasing it via their exosomes. OncomiR155 target TERF-1 gene (a telomerase inhibitor) and increases the resistance towards DNA damage of the tumor cells, induced by cisplatin (Challagundla et al., 2015). Acidic TME also contribute to chemoresistance by altering cell cycle control and accelerating anti-apoptosis mechanisms, furthermore, EMT inducers also render drug resistance e.g. in metastatic HCT-8 colon cancer cells undergoing EMT showed decreased chemosensitivity (Zhang et al., 2018). MDR cells secrete greater amount of exosomes that drug sensitive cells (Yousafzai et al., 2018). Moreover, the expression of P-gp on the recipient cells is proportional to the release of exosomes from the resistant cells (Steinbichler et al., 2019). Evidences reveal that TDEs mediate drug resistance in cancer cells and more studies are required to identify the exosomal biomarkers responsible for it so that they can be targeted for therapy.

\section{Exosomes in anti-cancer therapy}

TDEs are of current interest because they carry proteins and nucleic acids from the parental cell and serving as potent diagnostic biomarkers (Table 1). Likely, under hypoxia, the overexpression of Rab5 and Rab7 induces tumor cell invasion in lung cancer, breast cancer, melanoma patients. Rab11 is a potent biomarker for colorectal and pancreatic cancer with poor prognosis. Flotilins, HSPs, ALIX protein and TSG101 are also potent diagnostic and prognostic biomarkers. TSG101 helps in assessing amount of secreted exosomes (Jalalian et al., 2019). These biomarkers can also be advantageous for the detection of secondary metastasis sites like GFP-tagged CD63 is utilized to detect the secondary metastatic site for breast cancer (Suetsugu et al., 2013).

Exosomes are implicated as a potent vector for drugs, immunotherapy, vaccination, adjuvant therapy and gene therapy (Table 3). It also possess innate biocompatibility, ability to suppress tumor gene expression by their cargoes and less therapeutics toxicity(Saari et al., 2015). Exosomes mediated drug delivery has gained access over liposomal approaches because of their smaller size, ubiquitous presence, greater shelflife and higher efficacy (Tomasetti et al., 2017). Moreover, liposomes can be recognized as foreign particle due to their synthetic nature (Batista and Melo, 2019). Hybrid nanoparticles generated by incubating exosomes with liposomes can help in unresolved treatment strategies e.g. delivery of CRISPR-Cas9 system to MSCs ( $\mathrm{Liu}$ and $\mathrm{Su}, 2019$ ). The immature DC derived exosomes exhibit low immunogenicity as the lack of surface markers and can be easily isolated from patient's peripheral blood (Liu and Su, 2019). Exosomes biogenesis and their uptake can be targeted for cancer therapy e.g. usage of diannexincan block the TDEs internalization (Tomasetti et al., 2017). Exosomes exhibit increased stability and structural deformities of the loaded drug in systemic circulation to show the better efficacy (Bunggulawa et al., 2018). Improvised chemosensitivity could be targeted by transducing respective miRNAs with the stromal cells e.g. ADSCs derived exosomes may possess miRNAs of interest that can modulate chemoresistant gene to confer chemosensitivity. Interestingly, paclitaxel loaded MSCs secrete exosomes with minimizes metastasis as reported (Steinbichler et al., 2019). A new aspect of biomimetics has been implemented in case of ovarian cancer where exosome based metastatic trap (M-Trap) device serve as preferential site for the tumor cells to adhere, thereby disturbing the communication of tumor cells with the TME (De La Fuente et al., 2015). Exosomes bound to RNA nanoparticles (like antibodies) called "aptamers" confirms specific delivery of siRNA to cancer cells e.g in breast cancer binds to EGFR, in colorectal cancer binds to folic acid receptor and in prostate cancer binds to PSMA and all these targets and suppress survivin in the cancer types, thereby inhibiting metastasis (Fengmei Pi1, 2, 3, 4, Daniel W. Binzel1, 2, 3, 4, Tae Jin Lee3, 5,£, Zhefeng Li1, 2, 3, 4, Meiyan Sun6, Piotr Rychahou7, Hui Li1, 2, 3, 4, Farzin Haque1, 2, 3, 4, Shaoying Wang1, 2, 3, 4, Carlo M. Croce3, 5, Bin Guo6, B. Mark Evers7, and Peixuan Guo1, 2, 2018).

Although exosomes are potent as diagnostic biomarkers and cancer therapeutics but the main challenge lies in the fact of isolation of the exosomes, contamination with the other microvesicles and shortening of lifespan of the exosomes once they are out the living habitat (Rajagopal and Harikumar, 2018). Monolithic silica microtips coupled with anti-CD9 antibody has been implemented for fast and increased isolation of exosomes 
from patient samples (Ueda et al., 2014). However, recent advances in technologies have propelled out more sophisticated ways for exosome isolation like use of microfluidics techniques like Ex-Chip or Exo-Screen system or ExoSearch etc (Rajagopal and Harikumar, 2018).

Concluding remarks

Exosomes have emerged out as critical functional mediators of TME and thus the review focuses the every important attributes of exosomes. Here we have tried to withhold a picture of how exosomes play a vital role in secondary metastasis of cancer and its role in progression and regression of cancer and its therapy. Exosomes have also been reported to exhibit anti-tumor properties as well as in therapeutic approaches. Depending on the cellular conditions and type, exosomes are directed to perform their specific functions. Thus, exosomes have emerged out as a double-edged sword which marks their presence in both promotion and demotion of cancer and thus spotlighted in recent researches.

\section{References:}

Arderiu, G., Peña, E., and Badimon, L. (2015). Angiogenic Microvascular Endothelial Cells Release Microparticles Rich in Tissue Factor That Promotes Postischemic Collateral Vessel Formation. Arterioscler. Thromb. Vasc. Biol. 35 : 348-357.

Aslan, C., Maralbashi, S., Salari, F., Kahroba, H., Sigaroodi, F., Kazemi, T., et al. (2019). Tumor-derived exosomes: Implication in angiogenesis and antiangiogenesis cancer therapy. J. Cell. Physiol.234 : 1688516903.

Batista, I.A., and Melo, S.A. (2019). Exosomes and the future of immunotherapy in pancreatic cancer. Int. J. Mol. Sci. 20 :

Blanc, L., and Vidal, M. (2018). New insights into the function of Rab GTPases in the context of exosomal secretion. Small GTPases $9: 95-106$.

Blavier, L., Lazaryev, A., Shi, X.H., Dorey, F.J., Shackleford, G.M., and DeClerck, Y.A. (2010). Stromelysin1 (MMP-3) is a target and a regulator of Wnt1-induced epithelial-mesenchymal transition (EMT). Cancer Biol. Ther. $10: 198-208$.

Bretz, N.P., Ridinger, J., Rupp, A.K., Rimbach, K., Keller, S., Rupp, C., et al. (2013). Body fluid exosomes promote secretion of inflammatory cytokines in monocytic cells via Toll-Like receptor signaling. J. Biol. Chem. 288 : 36691-36702.

Bunggulawa, E.J., Wang, W., Yin, T., Wang, N., Durkan, C., Wang, Y., et al. (2018). Recent advancements in the use of exosomes as drug delivery systems 06 Biological Sciences 0601 Biochemistry and Cell Biology. J. Nanobiotechnology $16: 1-13$.

Burke, M., Choksawangkarn, W., Edwards, N., Ostrand-Rosenberg, S., and Fenselau, C. (2014). Exosomes from myeloid-derived suppressor cells carry biologically active proteins. J. Proteome Res. 13 : 836-843.

Challagundla, K.B., Wise, P.M., Neviani, P., Chava, H., Murtadha, M., Xu, T., et al. (2015). ExosomeMediated Transfer of microRNAs Within the Tumor Microenvironment and Neuroblastoma Resistance to Chemotherapy. J. Natl. Cancer Inst. 107 : 1-13.

Chen, W., Xiao, M., Zhang, J., and Chen, W. (2018). M1-like tumor-associated macrophages activated by exosome-transferred THBS1 promote malignant migration in oral squamous cell carcinoma. J. Exp. Clin. Cancer Res. $37: 1-15$.

Chow, A., Zhou, W., Liu, L., Fong, M.Y., Champer, J., Haute, D. Van, et al. (2014). Macrophage immunomodulation by breast cancer-derived exosomes requires Toll-like receptor 2-mediated activation of NF- $\varkappa$ B. Sci. Rep. 4 :.

Christianson, H.C., Svensson, K.J., Kuppevelt, T.H. Van, Li, J.P., and Belting, M. (2013). Cancer cell exosomes depend on cell-surface heparan sulfate proteoglycans for their internalization and functional activity. 
Proc. Natl. Acad. Sci. U. S. A. 110 : 17380-17385.

Ciravolo, V., Huber, V., Ghedini, G.C., Venturelli, E., Bianchi, F., Campiglio, M., et al. (2012). Potential role of HER2-overexpressing exosomes in countering trastuzumab-based therapy. J. Cell. Physiol.227 : 658-667.

Cocucci, E., Racchetti, G., and Meldolesi, J. (2009). Shedding microvesicles: artefacts no more. Trends Cell Biol. $19: 43-51$.

Conigliaro, A., and Cicchini, C. (2018). Exosome-Mediated Signaling in Epithelial to Mesenchymal Transition and Tumor Progression. J. Clin. Med. $8: 26$.

Domenis, R., Cesselli, D., Toffoletto, B., Bourkoula, E., Caponnetto, F., Manini, I., et al. (2017). Systemic $\mathrm{T}$ cells immunosuppression of glioma stem cell-derived exosomes is mediated by monocytic myeloid-derived suppressor cells. PLoS One 12 : 1-23.

Feng, D., Zhao, W.L., Ye, Y.Y., Bai, X.C., Liu, R.Q., Chang, L.F., et al. (2010). Cellular internalization of exosomes occurs through phagocytosis. Traffic $11: 675-687$.

Fengmei Pi1, 2, 3, 4, Daniel W. Binzel1, 2, 3, 4, Tae Jin Lee3, 5,£, Zhefeng Li1, 2, 3, 4, Meiyan Sun6, Piotr Rychahou7, Hui Li1, 2, 3, 4, Farzin Haque1, 2, 3, 4, Shaoying Wang1, 2, 3, 4, Carlo M. Croce3, 5, Bin Guo6, B. Mark Evers7, and Peixuan Guo1, 2, U. (2018). Nanoparticle Orientation to Control RNA Loading and Ligand Display on Extracellular Vesicles for Cancer Regression. Nat Nanotechnol. Author Manuscript; Available PMC 2018 June 11. 176 : 139-148.

Fujiwara, T., Eguchi, T., Sogawa, C., Ono, K., Murakami, J., Ibaragi, S., et al. (2018). Carcinogenic epithelialmesenchymal transition initiated by oral cancer exosomes is inhibited by anti-EGFR antibody cetuximab. Oral Oncol. $86: 251-257$.

Gangoda, L., Boukouris, S., Liem, M., Kalra, H., and Mathivanan, S. (2015). Extracellular vesicles including exosomes are mediators of signal transduction: Are they protective or pathogenic? Proteomics15 : 260-271.

Giallombardo, M., Taverna, S., Alessandro, R., Hong, D., and Rolfo, C. (2016). Exosome-mediated drug resistance in cancer: The near future is here. Ther. Adv. Med. Oncol. $8: 320-322$.

Gonda, A., Kabagwira, J., Senthil, G.N., and Wall, N.R. (2019). Internalization of exosomes through receptormediated endocytosis. Mol. Cancer Res. 17: 337-347.

Gonzalez-Avila, G., Sommer, B., Mendoza-Posada, D.A., Ramos, C., Garcia-Hernandez, A.A., and FalfanValencia, R. (2019). Matrix metalloproteinases participation in the metastatic process and their diagnostic and therapeutic applications in cancer. Crit. Rev. Oncol. Hematol. 137 : 57-83.

Greening, D.W., Gopal, S.K., Mathias, R.A., Liu, L., Sheng, J., Zhu, H.J., et al. (2015a). Emerging roles of exosomes during epithelial-mesenchymal transition and cancer progression. Semin. Cell Dev. Biol. 40 : $60-71$.

Greening, D.W., Gopal, S.K., Xu, R., Simpson, R.J., and Chen, W. (2015b). Exosomes and their roles in immune regulation and cancer. Semin. Cell Dev. Biol. $40: 72-81$.

Guo, Y., Ji, X., Liu, J., Fan, D., Zhou, Q., Chen, C., et al. (2019). Effects of exosomes on pre-metastatic niche formation in tumors. Mol. Cancer $18: 1-11$.

Haderk, F., Schulz, R., Iskar, M., Cid, L.L., Worst, T., Willmund, K. V., et al. (2017). Tumor-derived exosomes modulate PD-L1 expression in monocytes. Sci. Immunol. 2 : 1-12.

Heerboth, S., Housman, G., Leary, M., Longacre, M., Byler, S., Lapinska, K., et al. (2015). EMT and tumor metastasis. Clin. Transl. Med.4 :.

Henne, W.M., Buchkovich, N.J., and Emr, S.D. (2011). The ESCRT Pathway. Dev. Cell 21 : 77-91. 
Hessvik, N.P., and Llorente, A. (2018). Current knowledge on exosome biogenesis and release. Cell. Mol. Life Sci. 75 : 193-208.

Hristov, M., Erl, W., Linder, S., and Weber, P.C. (2004). Apoptotic bodies from endothelial cells enhance the number and initiate the differentiation of human endothelial progenitor cells in vitro. Blood 104 : 2761-2766.

Huang, T., and Deng, C.X. (2019). Current progresses of exosomes as cancer diagnostic and prognostic biomarkers. Int. J. Biol. Sci.15 : 1-11.

Huotari, J., and Helenius, A. (2011). Endosome maturation. EMBO J.30 : 3481-3500.

Jalalian, S.H., Ramezani, M., Jalalian, S.A., Abnous, K., and Taghdisi, S.M. (2019). Exosomes, new biomarkers in early cancer detection. Anal. Biochem. 571:1-13.

Kajimoto, T., Okada, T., Miya, S., Zhang, L., and Nakamura, S.I. (2013). Ongoing activation of sphingosine 1-phosphate receptors mediates maturation of exosomal multivesicular endosomes. Nat. Commun. 4 : 1-13.

Kevin Range, and D.M.Y.A.M. (2012). 基因的改变NIH Public Access. Bone23 : 1-7.

Kim, Y.S., Ahn, J.S., Kim, S., Kim, H.J., Kim, S.H., and Kang, J.S. (2018). The potential theragnostic (diagnostic+therapeutic) application of exosomes in diverse biomedical fields.

Korean J. Physiol. Pharmacol. 22 : 113-125.

La Fuente, A. De, Alonso-Alconada, L., Costa, C., Cueva, J., Garcia-Caballero, T., Lopez-Lopez, R., et al. (2015). M-Trap: Exosome-Based Capture of Tumor Cells as a New Technology in Peritoneal Metastasis. J. Natl. Cancer Inst. 107: 1-10.

Li, W., Li, C., Zhou, T., Liu, X., Liu, X., Li, X., et al. (2017). Role of exosomal proteins in cancer diagnosis. Mol. Cancer $16: 1-12$.

Lingwood, D., and Simons, K. (2010). Lipid rafts as a membrane-organizing principle. Science (80-. ). 327 : 46-50.

Liu, C., and Su, C. (2019). Design strategies and application progress of therapeutic exosomes. Theranostics $9: 1015-1028$.

Ludwig, A.K., and Giebel, B. (2012). Exosomes: Small vesicles participating in intercellular communication. Int. J. Biochem. Cell Biol. 44 : 11-15.

Maia, J., Caja, S., Strano Moraes, M.C., Couto, N., and Costa-Silva, B. (2018). Exosome-based cell-cell communication in the tumor microenvironment. Front. Cell Dev. Biol. $6: 1-19$.

Mashouri, L., Yousefi, H., Aref, A.R., Ahadi, A.M., Molaei, F., and Alahari, S.K. (2019). Exosomes: Composition, biogenesis, and mechanisms in cancer metastasis and drug resistance. Mol. Cancer 18 : 1-14.

Mathivanan, S., Ji, H., and Simpson, R.J. (2010). Exosomes: Extracellular organelles important in intercellular communication. J. Proteomics 73 : 1907-1920.

Maxson \& Mitchell (2016). 乳鼠心肌提取 HHS Public Access. Physiol. Behav. 176 : 139-148.

McGough, I.J., and Vincent, J.P. (2016). Exosomes in developmental signalling. Dev. 143 : 2482-2493.

McKelvey, K.J., Powell, K.L., Ashton, A.W., Morris, J.M., and McCracken, S.A. (2015). Exosomes: Mechanisms of Uptake. J. Circ. Biomarkers4 : 1-9.

Meng, Y., Sun, J., Wang, X., Hu, T., Ma, Y., Kong, C., et al. (2019). Exosomes: A promising avenue for the diagnosis of breast cancer. Technol. Cancer Res. Treat. $18: 1-14$.

Mulcahy, L.A., Pink, R.C., and Carter, D.R.F. (2014). Routes and mechanisms of extracellular vesicle uptake. J. Extracell. Vesicles3 : 1-14. 
Ochieng, J., Nangami, G., Sakwe, A., Rana, T., Ingram, S., Goodwin, J.S., et al. (2018). Extracellular histones are the ligands for the uptake of exosomes and hydroxyapatite-nanoparticles by tumor cells via syndecan-4. FEBS Lett. $592: 3274-3285$.

Ostrowski, M., Carmo, N.B., Krumeich, S., Fanget, I., Raposo, G., Savina, A., et al. (2010). Rab27a and Rab27b control different steps of the exosome secretion pathway. Nat. Cell Biol. 12 : 19-30.

Othman, N., Jamal, R., and Abu, N. (2019). Cancer-Derived Exosomes as Effectors of Key InflammationRelated Players. Front. Immunol.10 : 1-13.

Purushothaman, A., Bandari, S.K., Liu, J., Mobley, J.A., Brown, E.A., and Sanderson, R.D. (2016). Fibronectin on the surface of myeloma cell-derived exosomes mediates exosome-cell interactions. J. Biol. Chem.291: 1652-1663.

Rajagopal, C., and Harikumar, K.B. (2018). The origin and functions of exosomes in cancer. Front. Oncol. $8:$

Reclusa, P., Taverna, S., Pucci, M., Durendez, E., Calabuig, S., Manca, P., et al. (2017). Exosomes as diagnostic and predictive biomarkers in lung cancer. J. Thorac. Dis. 9 : S1373-S1382.

Record, M., Poirot, M., and Silvente-Poirot, S. (2014). Emerging concepts on the role of exosomes in lipid metabolic diseases. Biochimie $96: 67-74$.

Saari, H., Lázaro-Ibáñez, E., Viitala, T., Vuorimaa-Laukkanen, E., Siljander, P., and Yliperttula, M. (2015). Microvesicle- and exosome-mediated drug delivery enhances the cytotoxicity of Paclitaxel in autologous prostate cancer cells. J. Control. Release 220 : 727-737.

Safaei, R., Larson, B.J., Cheng, T.C., Gibson, M.A., Otani, S., Naerdemann, W., et al. (2005). Abnormal lysosomal trafficking and enhanced exosomal export of cisplatin in drug-resistant human ovarian carcinoma cells. Mol. Cancer Ther. 4 : 1595-1604.

Sheldon, H., Heikamp, E., Turley, H., Dragovic, R., Thomas, P., Oon, C.E., et al. (2010). New mechanism for Notch signaling to endothelium at a distance by delta-like 4 incorporation into exosomes. Blood 116 : 2385-2394.

Sinha, S., Hoshino, D., Hong, N.H., Kirkbride, K.C., Grega-Larson, N.E., Seiki, M., et al. (2016). Cortactin promotes exosome secretion by controlling branched actin dynamics. J. Cell Biol. 214 : 197-213.

Steinbichler, T.B., Dudás, J., Skvortsov, S., Ganswindt, U., Riechelmann, H., and Skvortsova, I.I. (2019). Therapy resistance mediated by exosomes. Mol. Cancer $18: 1-11$.

Stuffers, S., Sem Wegner, C., Stenmark, H., and Brech, A. (2009). Multivesicular endosome biogenesis in the absence of ESCRTs. Traffic10 : 925-937.

Su, M.-J., Parayath, N.N., and Amiji, M.M. (2018). Exosome-Mediated Communication in the Tumor Microenvironment (Elsevier Inc.).

Suetsugu, A., Honma, K., Saji, S., Moriwaki, H., Ochiya, T., and Hoffman, R.M. (2013). Imaging exosome transfer from breast cancer cells to stroma at metastatic sites in orthotopic nude-mouse models. Adv. Drug Deliv. Rev. $65: 383-390$.

Sumrin, A., Moazzam, S., Khan, A.A., Ramzan, I., Batool, Z., Kaleem, S., et al. (2018). Exosomes as biomarker of cancer. Brazilian Arch. Biol. Technol. 61 : 1-14.

Syn, N., Wang, L., Sethi, G., Thiery, J.P., and Goh, B.C. (2016). Exosome-Mediated Metastasis: From Epithelial-Mesenchymal Transition to Escape from Immunosurveillance. Trends Pharmacol. Sci. 37 : 606617.

Théry, C., Ostrowski, M., and Segura, E. (2009). Membrane vesicles as conveyors of immune responses. Nat. Rev. Immunol. 9 : 581-593. 
Théry, C., Zitvogel, L., and Amigorena, S. (2002). Exosomes: Composition, biogenesis and function. Nat. Rev. Immunol. 2 : 569-579.

Tickner, J.A., Urquhart, A.J., Stephenson, S.A., Richard, D.J., and O'Byrne, K.J. (2014). Functions and therapeutic roles of exosomes in cancer. Front. Oncol. $4 M A Y: 2-9$.

Todorova, D., Simoncini, S., Lacroix, R., Sabatier, F., and Dignat-George, F. (2017). Extracellular vesicles in angiogenesis. Circ. Res. $120: 1658-1673$.

Tomasetti, M., Lee, W., Santarelli, L., and Neuzil, J. (2017). Exosome-derived microRNAs in cancer metabolism: Possible implications in cancer diagnostics and therapy. Exp. Mol. Med. 49 : e285-11.

Trajkovic, K., Hsu, C., Chiantia, S., Rajendran, L., Wenzel, D., Wieland, F., et al. (2008). Ceramide triggers budding of exosome vesicles into multivesicular endosomes. Science (80-. ). 319 : 1244-1247.

Trams, E.G., Lauter, C.J., Norman Salem, J., and Heine, U. (1981). Exfoliation of membrane ecto-enzymes in the form of micro-vesicles. BBA - Biomembr. 645 : 63-70.

Ueda, K., Ishikawa, N., Tatsuguchi, A., Saichi, N., Fujii, R., and Nakagawa, H. (2014). Antibody-coupled monolithic silica microtips for highthroughput molecular profiling of circulating exosomes. Sci. Rep.4 : 1-9.

Vella, L.J. (2014a). The emerging role of exosomes in epithelial-mesenchymal-transition in cancer. Front. Oncol. $4: 1-6$.

Verma, M., Lam, T.K., Hebert, E., and Divi, R.L. (2015). Extracellular vesicles: Potential applications in cancer diagnosis, prognosis, and epidemiology. BMC Clin. Pathol. 15 : 1-9.

Villarroya-Beltri, C., Baixauli, F., Mittelbrunn, M., Fernández-Delgado, I., Torralba, D., Moreno-Gonzalo, O., et al. (2016). ISGylation controls exosome secretion by promoting lysosomal degradation of MVB proteins. Nat. Commun. 7 :.

Villarroya-Beltri, C., Gutiérrez-Vázquez, C., Sánchez-Cabo, F., Pérez-Hernández, D., Vázquez, J., MartinCofreces, N., et al. (2013). Sumoylated hnRNPA2B1 controls the sorting of miRNAs into exosomes through binding to specific motifs. Nat. Commun. 4 : 1-10.

Wan, Z., Gao, X., Dong, Y., Zhao, Y., Chen, X., Yang, G., et al. (2018). Exosome-mediated cell-cell communication in tumor progression. Am. J. Cancer Res. $8: 1661-1673$.

Wang, J., Liu, Y., Sun, W., Zhang, Q., Gu, T., and Li, G. (2018a). Plasma exosomes as novel biomarker for the early diagnosis of gastric cancer. Cancer Biomarkers $21: 805-812$.

Wang, X., Luo, G., Zhang, K., Cao, J., Huang, C., Jiang, T., et al. (2018b). Hypoxic tumor-derived exosomal miR-301a mediates M2 macrophage polarization via PTEN/PI3Kg to promote pancreatic cancer metastasis. Cancer Res. $78: 4586-4598$.

Webber, J., Yeung, V., and Clayton, A. (2015). Extracellular vesicles as modulators of the cancer microenvironment. Semin. Cell Dev. Biol.40 : 27-34.

Whiteside, T.L. (2015). The potential of tumor-derived exosomes for noninvasive cancer monitoring. Expert Rev. Mol. Diagn. 15 : 1293-1310.

Williams, R.L., and Urbé, S. (2007). The emerging shape of the ESCRT machinery. Nat. Rev. Mol. Cell Biol. $8: 355-368$.

Xu, J., Camfield, R., and Gorski, S.M. (2018). The interplay between exosomes and autophagy - partners in crime. J. Cell Sci. 131 : 1-11.

Yousafzai, N.A., Wang, H., Wang, Z., Zhu, Y., Zhu, L., Jin, H., et al. (2018). Exosome mediated multidrug resistance in cancer. Am. J. Cancer Res. $8: 2210-2226$. 
Yuan, J. hang, Yang, F., Wang, F., Ma, J. zhao, Guo, Y. jun, Tao, Q. fei, et al. (2014). A Long Noncoding RNA Activated by TGF- $\beta$ promotes the invasion-metastasis cascade in hepatocellular carcinoma. Cancer Cell25 : 666-681.

Zhang, C., Ji, Q., Yang, Y., Li, Q., and Wang, Z. (2018). Exosome: Function and role in cancer metastasis and drug resistance. Technol. Cancer Res. Treat. $17: 1-12$.

Zhang, H., Deng, T., Liu, R., Bai, M., Zhou, L., Wang, X., et al. (2017). Exosome-delivered EGFR regulates liver microenvironment to promote gastric cancer liver metastasis. Nat. Commun. 8 :.

Zorrilla, S.R., García, A.G., Carrión, A.B., Vila, P.G., Martín, M.S., Torreira, M.G., et al. (2019). Exosomes in head and neck cancer. Updating and revisiting. J. Enzyme Inhib. Med. Chem. 34 : 1641-1651.

Figure 1. Exosomes biogenesis

Figure 2. Role of exosomes in secondary metastasis

Figure 3. Dual role of exosomes in immune modulation

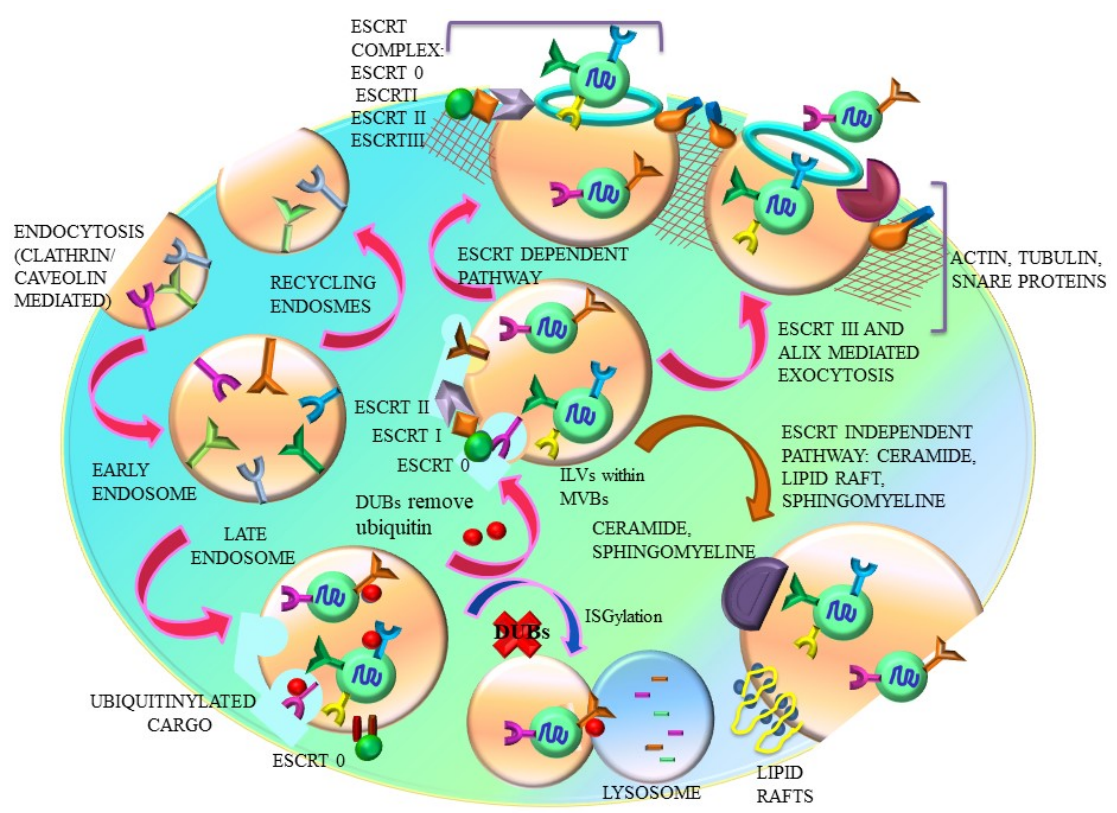





\section{Hosted file}

Table 1.docx available at https://authorea.com/users/333134/articles/459393-dynamicity-ofexosomes-as-immuno-oncological-biomarkers-in-secondary-metastasis-and-cancer-therapy

\section{Hosted file}

Table 2.docx available at https://authorea.com/users/333134/articles/459393-dynamicity-ofexosomes-as-immuno-oncological-biomarkers-in-secondary-metastasis-and-cancer-therapy 